\title{
Gene mutation defends against Alzheimer's disease
}

\section{Rare genetic variant suggests a cause and treatment for cognitive decline.}

\section{BY EWEN CALLAWAY}

A lmost 30 million people live with Alzheimer's disease worldwide, a staggering health-care burden that is expected to quadruple by 2050 . Yet doctors can offer no effective treatment, and scientists have not been able definitively to pin down the underlying mechanism of the disease.

Research published this week offers some hope on both counts, by showing that a lucky few people carry a genetic mutation that naturally prevents them from developing the condition ${ }^{1}$. The discovery not only confirms the principal suspect that is responsible for Alzheimer's, it also suggests that the disease could be an extreme form of the cognitive decline seen in many older people. The mutation - the first ever found to protect against the disease - lies in a gene that produces amyloid- $\beta$ precursor protein (APP), which has an unknown role in the brain and has long been suspected to be at the heart of Alzheimer's.

APP was discovered 25 years ago in patients with rare, inherited forms of Alzheimer's that strike in middle age $e^{2-5}$. In the brain, APP is broken down into a smaller molecule called amyloid- $\beta$. Visible clumps, or plaques, of amyloid- $\beta$ found in the autopsied brains of patients are a hallmark of Alzheimer's, but scientists have long debated whether the plaques are a cause of the neurodegenerative condition or a consequence of other biochemical changes associated with the disease. The latest finding supports other genetics studies blaming amyloid- $\beta$, and it makes the protein "the prime therapeutic target”, says Rudolph Tanzi, a neurologist at the Massachusetts General Hospital in Boston and a member of one of the four teams that discovered APP's role in the 1980s.

If amyloid- $\beta$ plaques were confirmed as the cause of Alzheimer's, it would bolster efforts to develop drugs that block their formation in order to treat or prevent the ravaging condition, says Kári Stefánsson, chief executive of deCODE Genetics in Reykjavik, Iceland, who led the latest research. He and his team first discovered the mutation by comparing the complete genome sequences of 1,795 Icelanders with their medical histories. The researchers then studied the variant in nearly 400,000 more Scandinavians.

The variant is rare, but it has a huge impact on those fortunate enough to inherit even a single copy of it. About $0.5 \%$ of Icelanders are carriers, as are $0.2-0.5 \%$ of Finns, Swedes and Norwegians. Compared with their countrymen who lack the mutation, Icelanders who carry it are more than five times more likely to reach 85 without being diagnosed with Alzheimer's.

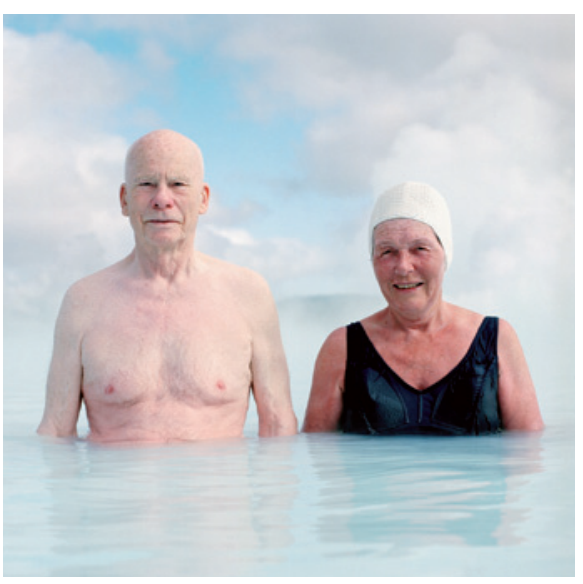

About $0.5 \%$ of Icelanders have a protective gene that prevents mental deterioration in old age.

They also live longer, with a $50 \%$ better chance of celebrating their 85 th birthday.

The mutation seems to put a brake on the milder mental deterioration that most elderly people experience. Carriers are about 7.5 times more likely than non-carriers to reach the age of 85 without suffering major cognitive decline, such as memory loss. They also perform better on the cognitive tests that are administered thrice yearly to Icelanders who live in nursing homes.

For Stefánsson, this suggests that Alzheimer's disease and cognitive decline are two sides of the same coin, with a common cause - the build-up of amyloid- $\beta$ plaques in the brain, something seen to a lesser degree in elderly people who do not develop full-blown Alzheimer's. "Pathologists have always suspected that there was a substantial overlap between Alzheimer's disease and normal agerelated changes," says Stefánsson. A drug that mimics the effects of the mutation, he says, would have the potential both to slow cognitive decline and to prevent Alzheimer's.

Stefánsson and his team discovered that the mutation introduces a single amino-acid alteration to APP. This amino acid is close to the site where an enzyme called $\beta$-secretase 1 (BACE1) ordinarily snips APP into smaller amyloid- $\beta$ chunks - and the alteration is enough to reduce the enzyme's efficiency.

Blocking BACE1 to treat Alzheimer's is not a new idea. Drug companies have been working on 'BACE inhibitors' for more than a decade, and several are now in clinical trials. Stefánsson's study suggests that blocking $\beta$-secretase from cleaving APP does indeed have the potential to prevent Alzheimer's, says Philippe Amouyel, an epidemiologist at the Pasteur Institute in Lille, France. But "it is very difficult to identify the precise time when this amyloid toxic effect could still be modified", he warns. "If this effect needs to be blocked as early as possible in life to protect against Alzheimer's disease, we will need to propose a new design for clinical trials" to identify an effective treatment.

Julie Williams, an expert on the genetics of Alzheimer's disease at Cardiff University, UK, agrees that amyloid- $\beta$ is strongly implicated by the latest research, but adds that “it still doesn't say it's the only factor we should be targeting, in terms of therapies".

For Stefánsson, the results are also a powerful demonstration that whole-genome sequencing can uncover very rare mutations that nonetheless offer insight into common diseases. He argues that most human differences, including disease risk, are determined by common genetic variants that each tilt the odds of developing a disease only slightly.

Rarer mutations, by contrast, tend to skew someone's disease risk much more strongly, but only in a handful of patients. "The rare variants are not going to explain a large amount [of disease], but they are going to provide very key mechanistic insights into how all of this happens," says Stefánsson. He and his team will soon publish studies on rare variants that influence the risk of other conditions, including ovarian cancer and gout. "We are going to see a lot of these," he says.

\footnotetext{
1. Jonsson, T. et al. Nature http://dx.doi.org/10.1038/ nature11283 (2012).

2. Kang, J. et al. Nature $\mathbf{3 2 5}$, 733-736 (1987)

3. Goldgaber, D., Lerman, M. I., McBride, O. W., Saffiotti, U. \& Gajdusek, D. C. Science 235, 877-880 (1987).

4. Robakis, N. K., Ramakrishna, N., Wolfe, G. \& Wisniewski, H. M. Proc. Natl Acad. Sci. USA 84, 4190-4194 (1987).

5. Tanzi, R. E. et al. Science 235, 880-884 (1987).
} 